\section{ORIGINAL RESEARCH}

J.W. Lee

E.Y. Cho

S.H. Hong

H.W. Chung

J.H. Kim

K.-H. Chang

J.-Y. Choi

J.-S. Yeom

H.S. Kang

\title{
Spinal Epidural Hemangiomas: Various Types of MR Imaging Features with Histopathologic Correlation
}

BACKGROUND AND PURPOSE: Because of the high vascularization of hemangiomas, preoperative misinterpretation may result in unexpected intraoperative hemorrhage and incomplete resection, which results in the persistence of clinical symptoms or recurrence. Our purpose was to analyze various MR imaging features of a spinal epidural hemangioma with histopathologic correlation.

MATERIALS AND METHODS: After searching through the pathology data bases in 3 hospitals, we included 14 patients (9 male and 5 female; mean age, 38 years; age range, 2-62 years) with spinal epidural hemangiomas confirmed by surgical resection after MR imaging. Three radiologists reviewed the MR imaging in consensus and categorized the features into subtypes on the basis of histopathologic findings.

RESULTS: We categorized the MR imaging features as follows: type A for a cystlike mass with T1 hyperintensity ( 2 cases, arteriovenous type with an organized hematoma), type B for a cystlike mass with $\mathrm{T} 1$ isointensity ( 3 cases, venous type), type $\mathrm{C}$ for a solid hypervascular mass ( 7 cases, cavernous type), and type D for an epidural hematoma (2 cases, cavernous type with hematoma). Types $A$ and $B$ had frequent single segmental involvement $(4 / 5)$, whereas types $C$ and $D$ had multisegmental involvement in all. Regardless of MR types, lobular contour (8/14) and a rim of low T2 signal intensity (8/14) of the mass were common. T1 hyperintensity of the mass was occasionally seen (5/14).

CONCLUSIONS: Spinal epidural hemangiomas can have various MR imaging features according to their different histopathologic backgrounds. In addition to common features such as solid hypervascularity, lobular contour, and a rim of low T2 signal intensity, T1 hyperintensity or multisegmental involvement may also be a clue in the differential diagnosis of a spinal epidural hemangioma.

$\mathbf{H}$ emangiomas are not considered to be vascular neoplasms, but rather hamartomas or malformation of the microcirculation. They are classified by the predominant type of vascular channel (capillary, cavernous, arteriovenous, or venous). In the spine, hemangiomas located in the epidural space are rare. Most of the spinal epidural hemangiomas reported previously were of the cavernous type. They constitute approximately $4 \%$ of all epidural tumors and $12 \%$ of all intraspinal hemangiomas. ${ }^{1-13}$

There have been several reports about MR features of a spinal epidural hemangioma. ${ }^{4-6,8,9,11-13}$ However, most are case reports about the cavernous type. We have found only 2 original articles that describe the MR imaging features of cavernous hemangiomas (with only 5 cases in each article). ${ }^{8,9}$ According to the 2 studies, the cavernous spinal epidural hemangiomas showed a lobular epidural mass; high T2 signal intensity; and homogeneous, strong enhancement on MR images. Recently, we have experienced various MR imaging features of epidural hemangiomas that are somewhat different

Received November 14, 2006; accepted after revision December 17.

From the Departments of Radiology (J.W.L., J.H.K., H.S.K.) and Orthopaedic Surgery (J.-S.Y.), Seoul National University Bundang Hospital, Gyeongi-Do, Korea; Departments of Pathology (E.Y.C.) and Radiology and Center for Imaging Science (H.W.C.), Samsung Medical Center, Sungkyunkwan University School of Medicine, Seoul, Korea; and the Department of Radiology and Institute of Radiation Medicine (S.H.H., K.-H.C., J.-Y.C.), Seoul National University College of Medicine, Seoul, Korea.

Address correspondence to Eun Yoon Cho, MD, Department of Pathology, Samsung Medical Center, 50 Ilwon-Dong, Kangnam-gu, Seoul 135-710, Korea; e-mail: eycmd@ lycos.co.kr

Indicates article with supplemental on-line tables.

DOI 10.3174/ajnr.A0563 from those of previous reports and with various histopathologic backgrounds.

The differential diagnosis for spinal epidural hemangiomas before surgical resection included schwannoma, lymphoma, meningioma, angiolipoma, disk herniation, synovial cysts, granulomatous infection, pure epidural hematoma, and extramedullary hematopoiesis. ${ }^{5,8,11,14-19}$ Because of the high vascularization of hemangiomas, this misinterpretation may result in unexpected intraoperative hemorrhage. Incomplete surgical removal of a spinal epidural hemangioma because of diffuse bleeding or minimal exposure during disk surgery might result in the persistence of clinical symptoms or recurrence. Reoperation for remnant or recurrent epidural hemangioma is very difficult because of peridural or periradicular adhesion and unclear tumor margins; as a result, complete resection cannot be guaranteed in reoperation..$^{5,9,11}$ Therefore, proper preoperative planning and complete resection in the first operation is essential. For this, preoperative suspicion of epidural hemangioma is important. The purpose of this study was to analyze MR imaging features of a spinal epidural hemangioma with histopathologic correlation.

\section{Materials and Methods}

\section{Patients}

Our institutional review board approved this study, and informed consent was not required. After a search of all available electronic pathologic data bases in 3 hospitals (6 years in 2 hospitals, 3 years in 1 hospital) by 3 radiologists, cases confirmed as spinal epidural hemangiomas after open surgical resections and preoperative MR images were included in this study. Vertebral body hemangiomas with epidural extension were excluded. One radiologist reviewed medical 
records for age, sex, chief complaints, and duration of symptoms. The clinical features are summarized in On-line Table 1. Fourteen patients ( 9 male, 5 female; mean age, 38 years; age range, 2-62 years) were included in this study. MR was done in all cases of spinal epidural hemangioma before surgical resection, so there was no case that was excluded as a result of the absence of preoperative MR. Radiculopathy were in 7 , myelopathy in 6 , and nonspecific axial pain in 1 . Mean duration of symptoms was approximately 555 days (range, 2 days to 8 years). There was no case of incomplete resection of the hemangioma and no case of persistence or recurrence after the operation. Follow-up was done at a mean duration of 647 days (range, 125-1416 days).

\section{MR Imaging}

MR imaging was performed by various MR scanners with the use of different protocols at diverse hospitals before referral to the 3 hospitals. Most cases were imaged with the use of phased array spine coil and a $1.5 \mathrm{~T}$ superconducting magnet (Magnetom Vision, Siemens, Erlangen, Germany; Gyroscan Intera, Philips Medical Systems, Eindhoven, the Netherlands). Transverse and sagittal spin-echo T1- (TR, 250-700 ms; TE, 12-30 ms) and T2-weighted MR images (TR, 2000$5000 \mathrm{~ms}$; TE, 80-120 ms) were present in all cases. Contrastenhanced T1-weighted MR images after intravenous injection of gadopentetate dimeglumine $(0.1 \mathrm{mmol} / \mathrm{kg}$, Magnevist; Schering, Berlin, Germany) were present in all cases except for 1 .

\section{Analysis of MR Images}

Three radiologists retrospectively evaluated MR images in consensus. The radiologists had a total of 22 years of combined experience in spinal imaging. They were not blinded to their interpretations of the MR images. They focused on 1) signal intensity characteristics of the masses on T1- and T2-weighted, and contrast-enhanced images; 2) the location and level with craniocaudal extension; 3 ) the shape of the masses; 4) the presence of an associated hematoma; 5) mass effect such as cord compression, neural foraminal widening, change on adjacent bony vertebra; and 6) other findings such as the presence of dural tail sign and a rim of low signal intensity. ${ }^{3,8,9}$ Signal intensities of the masses on T1-weighted images were described as low, isointense, or high, relative to those of the intervertebral disk. Signal intensities of the masses on T2-weighted images were defined as bright high if the signal intensity of a mass was the same as the signal intensity in the CSF, high if the signal intensity was lower than that of the CSF and higher than that of muscle, intermediate if the signal intensity was the same as that of muscle, and low if lower than that of muscle. The signal intensities of the masses were also defined as homogeneous or heterogeneous. We regarded the mass as cystlike when it showed homogeneous bright high signal intensity or high signal intensity on a T2weighted image without homogeneous enhancement. If not, we regarded the mass as solid. Locations of masses were described as either anterior or posterior epidural space considering the predominant involvement. The level of craniocaudal extension was described as the number of vertebrae in which masses were present.

The contour of the mass was classified as smooth, lobular, or illdefined. ${ }^{8}$ If a mass had its own round or oval shape with clear demarcation, we considered it smooth contour. If a clearly demarcated mass was shaped by the adjacent anatomic structure and indented or dumbbell shaped, we considered it lobular contour. If we could not define the outer margin of the mass clearly, we considered it an illdefined contour. The presence of a hematoma was considered positive if there were characteristic MR signals suggestive of a hematoma.
A hematoma was considered present when there was very low signal intensity on the T2-weighed image and high signal intensity on the T1-weighted image. Very low signal intensity on the T2-weighted image and high signal intensity on the T1-weighted image were only seen for short periods of time during evolution of the hematoma. However, other signals such as isointensity or high signal intensity on a T2-weighted image and isointensity or low signal intensity on a T1-weighted image, which could be seen during evolution of the hematoma, were nonspecific and were seen in any mass in addition to a hematoma. Therefore, only those cases with a very low signal intensity on a T2-weighted image and a high signal intensity on a T1-weighted image were regarded as indicative of a hematoma. ${ }^{18}$

The dural tail sign was positive if there was thin dural enhancement near the mass with a broad angle. We evaluated a rim of low signal intensity on T1-, T2-, and contrast-enhanced MR images to check if there was a rim of low signal intensity surrounding the mass. During the assessment, 3 radiologists were blinded to pathologic details.

\section{Histopathologic Correlation of MR Imaging}

One pathologist reviewed all the pathologic slides, again without information concerning MR features, and described the histopathologic details. After the 3 radiologists who interpreted the images and the pathologist reached a consensus, they tried to categorize the MR imaging features into subtypes on the basis of the histopathologic findings.

\section{Results}

\section{MR Imaging Features}

The MR imaging features are summarized in Tables 1-3. There was an anterior epidural location in 5 cases: 4 at the lumbar spine, and 1 at the cervicothoracic junction. There was a posterior epidural location in 9 cases: 5 at the thoracic spine; and 4 at the cervical or cervicothoracic junction. All cases that were present in the lumbar or lumbosacral spine were located in the anterior epidural space. There were cystlike masses in 5 cases; bright high signal intensity with a clearly demarcated rim on T2-weighted images in 4; and high signal intensity on T2-weighted images in 1 . These 5 cases all showed peripheral enhancement. Among them, 2 cases showed high signal intensity on T1-weighted images and 3, isointensity. The other 9 cases showed a solid mass with strong enhancement (64\%, 9/14). Among the 9 cases, 2 showed MR features of a hematoma.

Considering the MR signal intensity characteristics, we categorized all cases into 4 types: type A for a cystlike mass with T1 hyperintensity (2 cases, Fig 1), type B for a cystlike mass with T1 isointensity ( 3 cases, Fig 2 ), type $\mathrm{C}$ for a solid hypervascular mass ( 7 cases, Fig 3 ), and type D for an epidural hematoma (2 cases, Fig 4). The anterior epidural space of the lumbar spine was a common location of types A and B (80\%, $4 / 5)$, whereas the posterior epidural space of the thoracic or cervical spine were common in types C and D (89\%, 8/9). Four of the 5 patients with type $\mathrm{A}$ and $\mathrm{B}$ lesions were young men (two 18-year-olds, one 20-year-old, and one 23-year-old). Types A and B showed single-segment spinal involvement except for 1 case, which showed 2 segments. However, all cases from types $\mathrm{C}$ and $\mathrm{D}$ showed multisegmental spinal involve- 


\begin{tabular}{|c|c|c|c|c|c|c|c|}
\hline Case No. & Location & Level & Epidural & T1WI & $\mathrm{T} 2 \mathrm{WI}$ & $\mathrm{CE}$ & MR Type \\
\hline 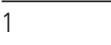 & L5-S1 & 1 & Ant & High & Bright high with rim & NA & A \\
\hline 2 & C7-T1 & 2 & Post & Iso & High with rim & Peripheral strong & B \\
\hline 3 & L5 & 1 & Ant & HT high & Bright high with rim & HT strong & A \\
\hline 4 & $\mathrm{~T} 11-\mathrm{T} 12$ & 2 & Post & Iso & HM high & HM strong & C \\
\hline 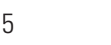 & T2-T4 & 4 & Post & Iso & HM bright high & HM strong & C \\
\hline 6 & T9-T11 & 4 & Post & Iso with bright foci & HT high & HM strong & C \\
\hline 7 & C6-T2 & 4 & Post & Iso & HM high & HM strong & C \\
\hline 3 & C5-T4 & 7 & Both & High & HT high and low & HT strong & $\mathrm{D}$ \\
\hline$y$ & T6-7 & 2 & Post & Iso & HM high & HM strong & C \\
\hline 10 & $\mathrm{C} 5-\mathrm{C} 7$ & 4 & Post & High with bright foci & HT high and low & HT strong & $D$ \\
\hline 11 & T3-T4 & 2 & Post & Iso & HM high & HM strong & C \\
\hline 12 & $\llcorner 4-5$ & 1 & Ant & Iso & Bright high with rim & Peripheral strong & $B$ \\
\hline 13 & L3 & 1 & Ant & Iso & Bright high with rim & Peripheral strong & B \\
\hline 14 & C6-T1 & 5 & Ant & Iso & HM high & HM strong & C \\
\hline
\end{tabular}

Note:- Level indicates craniocaudal extension described as the number of vertebrae where mass was present; Epidural, location of masses either anterior (Ant) or posterior (Post) epidural space; T1WI, T1-weighted MR image; T2WI, T2-weighted MR image; CE, contrast-enhanced MR image; A, cystlike mass with T1 hyperintensity; B, cystlike mass with T1 isointensity; C, solid hypervascular mass; D, epidural hematoma; Iso, isointense; HM, homogeneous signal intensity; HT, heterogeneous signal intensity; Peripheral, peripheral enhancement.

\begin{tabular}{|c|c|c|c|c|c|c|c|c|c|}
\hline $\begin{array}{l}\text { Case } \\
\text { No. }\end{array}$ & $\begin{array}{l}\text { MR } \\
\text { Type }\end{array}$ & Contour & $\begin{array}{c}\text { Low Signal } \\
\text { Rim on T1WI }\end{array}$ & $\begin{array}{l}\text { Low Signal } \\
\text { Rim on T2WI }\end{array}$ & $\begin{array}{l}\text { Low Signal } \\
\text { Rim on CE }\end{array}$ & $\begin{array}{l}\text { Dural Tail } \\
\text { Sign }\end{array}$ & $\begin{array}{l}\text { Cord } \\
\text { Comp }\end{array}$ & $\begin{array}{c}\mathrm{NF} \\
\text { Widen }\end{array}$ & $\begin{array}{c}\text { Vertebral } \\
\text { Body }\end{array}$ \\
\hline 1 & $A$ & Smooth & Yes & Yes & NA & NA & No & No & Intact \\
\hline 2 & B & Lobular & No & Yes & No & No & No & No & Intact \\
\hline 3 & A & Lobular & Yes & Yes & Yes & No & No & No & Intact \\
\hline 4 & $C$ & Smooth & Yes & Yes & No & Yes & No & No & Intact \\
\hline 5 & C & Lobular & No & No & No & No & No & Yes & Intact \\
\hline 6 & C & Lobular & No & No & No & Yes & Yes & Yes & Intact \\
\hline 7 & C & Lobular & Yes & Yes & No & Yes & Yes & Yes & Erosion \\
\hline 8 & D & III-defined & No & No & No & Yes & Yes & Yes & Intact \\
\hline 9 & C & Smooth & Yes & Yes & Yes & No & Yes & No & Intact \\
\hline 10 & D & III-defined & No & No & No & No & Yes & No & Intact \\
\hline 11 & C & Lobular & No & Yes & Yes & No & Yes & Yes & Erosion \\
\hline 12 & B & Lobular & No & Yes & No & No & No & No & Intact \\
\hline 13 & B & Smooth & No & No & No & No & No & No & Intact \\
\hline 14 & C & Lobular & Yes & Yes & No & No & Yes & Yes & Erosion \\
\hline
\end{tabular}

Note:-T1WI indicates T1-weighted MR image; T2WI, T2-weighted MR image; CE, contrast-enhanced MR image; Cord comp, cord compression; NF widen, neural foraminal widening; Vertebral body, involvement of vertebral body.

\begin{tabular}{|c|c|c|c|c|c|c|c|}
\hline $\begin{array}{l}\text { Case } \\
\text { No. }\end{array}$ & $\begin{array}{l}\text { MR } \\
\text { Type }\end{array}$ & $\begin{array}{l}\text { Histological } \\
\text { Feature }\end{array}$ & Hematoma & Location & Epidural & Level & $\mathrm{CC}$ \\
\hline 1 & A & Arteriovenous & Yes & Lumbosacral & Ant & 1 & Radiculopathy \\
\hline 2 & B & Venous & No & Cervicothoracic & Post & 2 & Radiculopathy \\
\hline 3 & A & Arteriovenous & Yes & Lumbar & Ant & 1 & Radiculopathy \\
\hline 4 & C & Cavernous & No & Thoracic & Post & 2 & Radiculopathy \\
\hline 5 & C & Cavernous & No & Thoracic & Post & 4 & Axial pain \\
\hline 6 & C & Cavernous & No & Thoracic & Post & 4 & Myelopathy \\
\hline 7 & C & Cavernous & No & Cervicothoracic & Post & 4 & Myelopathy \\
\hline 8 & D & Cavernous & Yes & Cervicothoracic & Post & 7 & Myelopathy \\
\hline 9 & C & Cavernous & No & Thoracic & Post & 2 & Myelopathy \\
\hline 10 & D & Cavernous & Yes & Cervical & Post & 4 & Radiculopathy \\
\hline 11 & C & Cavernous & No & Thoracic & Post & 2 & Myelopathy \\
\hline 12 & B & Venous & No & Lumbar & Ant & 1 & Radiculopathy \\
\hline 13 & B & Venous & No & Lumbar & Ant & 1 & Radiculopathy \\
\hline 14 & C & Cavernous & No & Cervicothoracic & Ant & 5 & Myelopathy \\
\hline
\end{tabular}

Note:-A indicates cystlike mass with T1 hyperintensity; B, cystlike mass with T1 isointensity; C, solid hypervascular mass; D, epidural hematoma; Epidural, location of masses either anterior (Ant) or posterior (Post) epidural space; Level, craniocaudal extension described as the number of vertebrae where mass was present; CC, chief complaint.

ment (range, 2-7 segment of spinal level; mean, 3.6 segment of spinal level).

A dural tail sign was positive in $4(28.6 \%)$ of 14 cases. There was compression of the spinal cord in 7 (50\%) of 14, neural foraminal widening in $6(43 \%)$ of 14 , and erosion of the ver- tebral body in $3(21 \%)$ of 14 . A dural tail sign, cord compression, neural foraminal widening, and vertebral erosions could be seen only in a cavernous mass (type C or D).

Regardless of MR types, lobular contour and a rim of low $\mathrm{T} 2$ signal intensity were relatively common. Lobular contours 

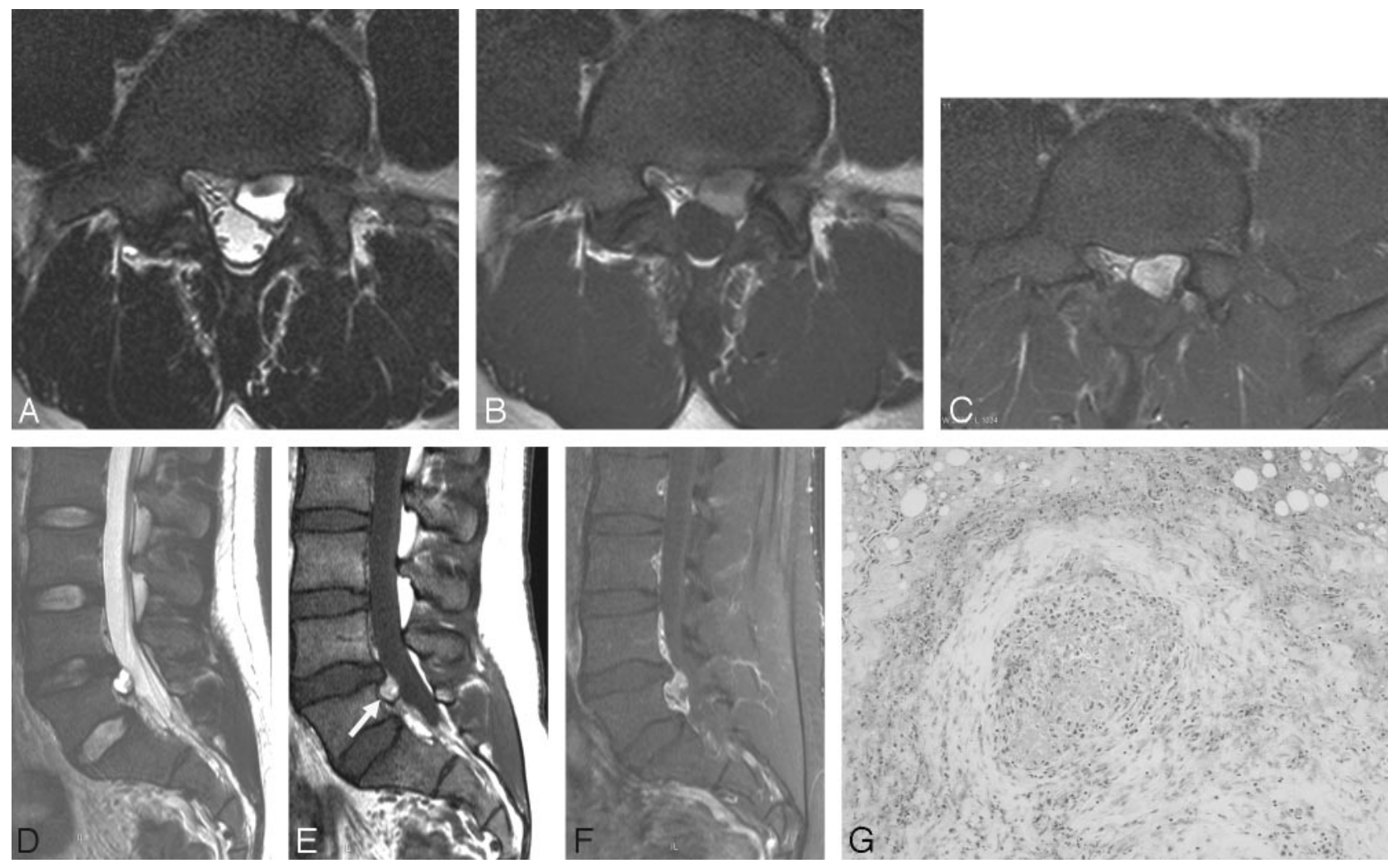

Fig 1. Case 3, a cystlike mass with $\mathrm{T} 1$ hyperintensity (type A).

A, The transverse T2-weighted spin-echo MR image demonstrates a well-defined mass at the anterior epidural space of L5. The mass shows bright high signal intensity, same as that of CSF. There is a rim of low signal intensity.

$B$, On the transverse noncontrast T1-weighted image, the mass shows heterogeneous high signal intensity with a rim of low signal intensity. Signal intensity of the anterior portion of the mass is lower than that of the posterior portion.

$C$, On the transverse fat-suppressed, postcontrast T1-weighted image, the anterior portion of the mass also shows strong enhancement.

$D$, On the sagittal T2-weighted image, the mass is located at the anterior epidural space of the L 5 spinal level. The mass shows bright high signal intensity with a rim of low signal intensity. $E$, On the sagittal noncontrast T1-weighted image, the mass (arrow) shows higher signal intensity than that of the intervertebral disk. We can see the rim of low signal intensity in the distal margin of the mass.

F, On the sagittal fat-suppressed, postcontrast T1-weighted image, the mass shows strong heterogeneous enhancement.

$G$, On microscopic examination, there is a vascular tumor composed of variable-sized, thick-walled, muscularized vessels, suggestive of an arteriovenous hemangioma. There is a focus of an organized hematoma with pigments of hemosiderin (H\&E, $\times 100)$.

were present in 8 of 14 cases (57\%, $1 / 2$ in type $A, 2 / 3$ in type $B$, $5 / 7$ in type C), smooth in 4 , and ill-defined in 2 (all type D). A rim of low T2 signal intensity was noted in 8 of the 14 cases ( $57 \%, 2 / 2$ in type $A, 2 / 3$ in type $B, 4 / 7$ in type C). Among them, a rim of low T1 signal intensity was also present in 6 and on contrast-enhanced images in 3 . The mass showed T1 hyperintensity in $5(35.7 \%)$ of the 14 cases.

\section{MR Imaging-Histopathologic Correlations}

Histopathologic correlations of MR imaging are summarized in Table 3. Histopathologically, type A lesions were arteriovenous hemangiomas with diffuse areas of organized hematoma caused by malfunctioning of the microcirculation. The area of the hematoma was composed of small capillary proliferation and fibrosis with infiltration of hemosiderin pigments and macrophages (Fig $1 G$ ). Type B lesions were hemangiomas composed of cystlike dilated thick blood vessels with smooth muscle, which suggested venous hemangioma. There was no hemorrhage (Fig 2E). Type C lesions demonstrated the typical histomorphologic features of cavernous hemangiomas with closely packed dilated ves- sels. The lesions were composed of thin-walled sinusoidal vascular spaces of varying sizes, which were surrounded by loose connective and adipose tissue, with no necrosis, hemorrhage, or degenerative change (Fig 3D). Type D lesions showed a diffuse, organized hematoma with focal areas of cavernous hemangioma consisting of sinusoidal vascular spaces lined by a thin fibrous wall (Fig $4 D$ ).

\section{Discussion}

Spinal epidural hemangiomas are extremely rare, and most are cavernous. ${ }^{1-13,20-23}$ According to our results and previous reports, the most common MR features of cavernous spinal epidural hemangiomas were solid hypervascular masses with lobular contour. There have been only 2 case reports describing MR features of other types of spinal epidural hemangiomas. ${ }^{3,13}$ In a report on 3 cases of spinal epidural hemangiomas by Rovira et al, ${ }^{13} 1$ case was of the arteriovenous type and was seen as a cystic mass on the MR image in the anterior epidural space of L4, which was similar to the findings of our study. The other case described a capillary hemangioma, which was seen as a solid hypervascular mass on MR. ${ }^{3}$ There was no previous 

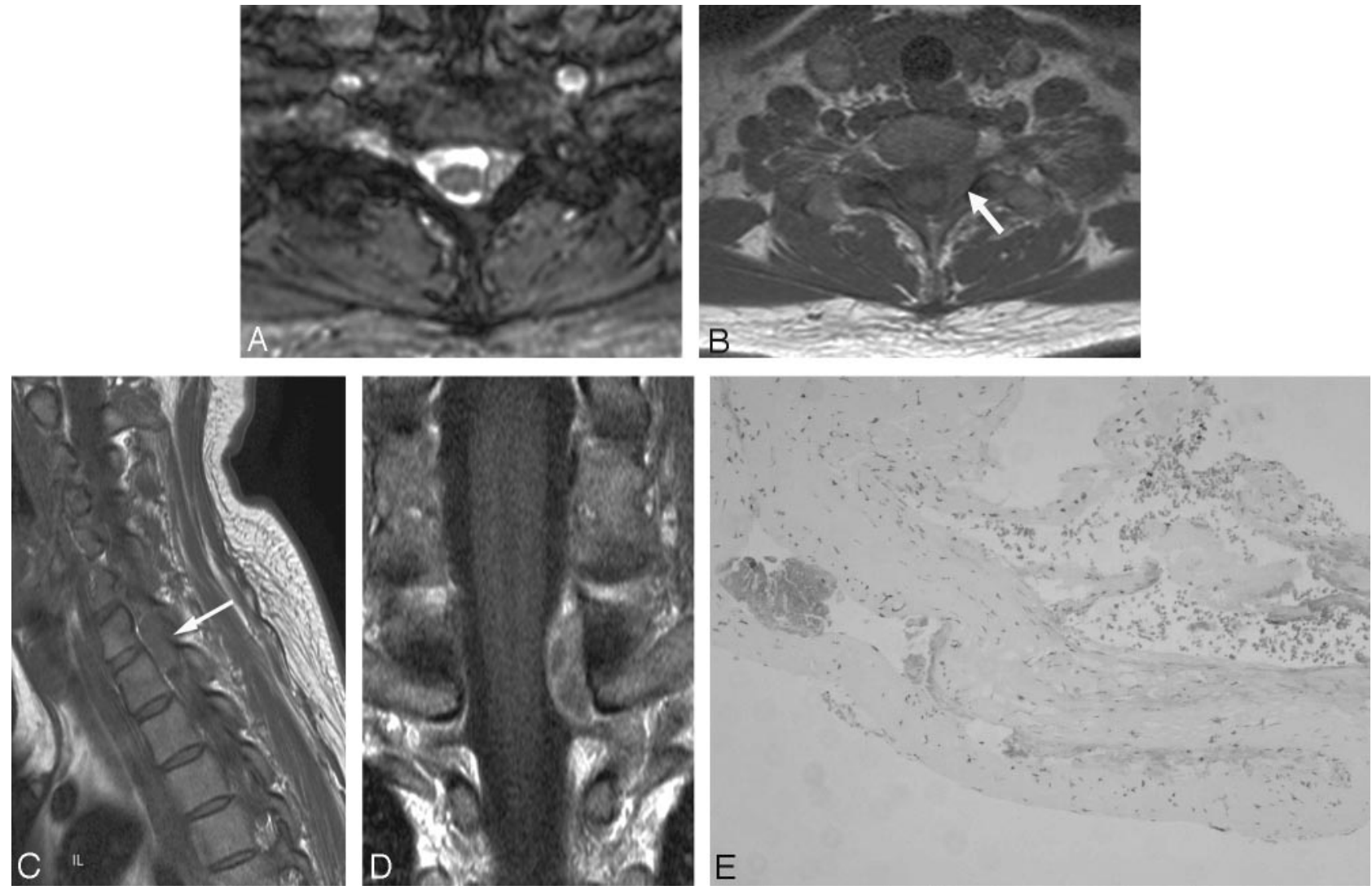

Fig 2. Case 2, a cystlike mass with $\mathrm{T} 1$ isointensity (type $\mathrm{B}$ ).

A, Transverse T2-weighted gradient-echo MR image demonstrates a well-defined mass with high signal intensity at the left posterior epidural space of C7-T1.

$B$, On transverse noncontrast T1-weighted MR image, the mass (arrow) shows homogeneous isointensity.

C, On sagittal noncontrast T1-weighted MR image, the signal intensity of the mass (arrow) is isointense to that of the intervertebral disk.

$D$, On coronal postcontrast T1-weighted image, the mass shows peripheral thick enhancement with inner septumlike enhancement.

E, Histologic section shows cystic dilated vessels with smooth muscle in the walls, suggestive of a venous hemangioma $(H \& E, \times 100)$. There is no evidence of an organized hematoma
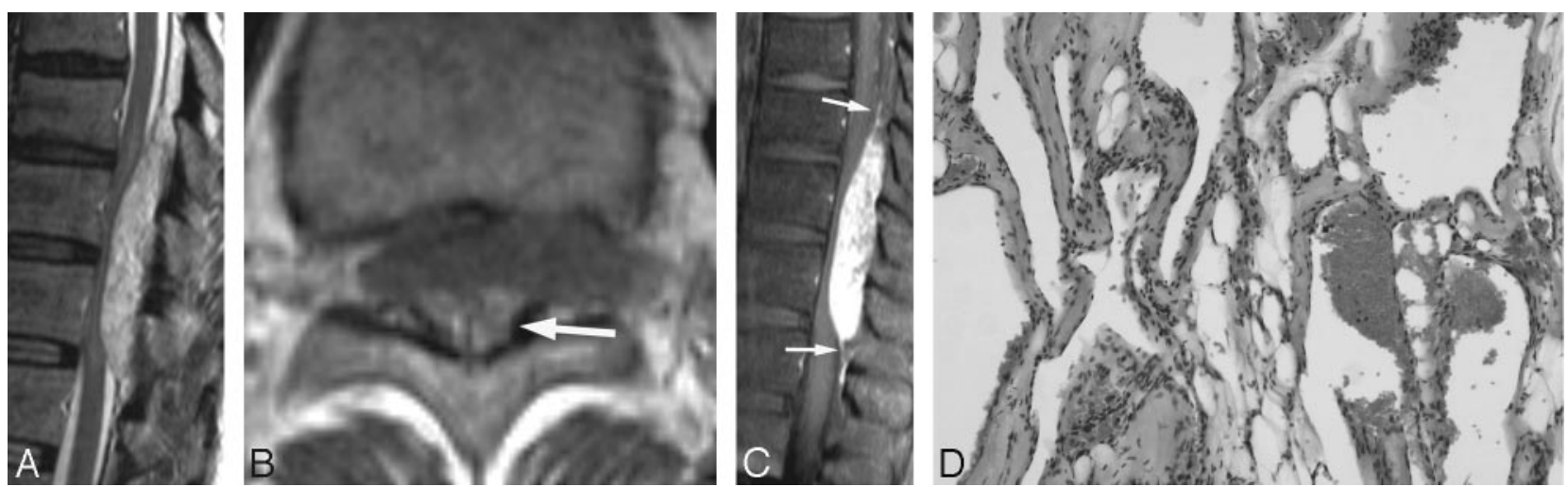

Fig 3. Case 6, a solid hypervascular mass (type C).

A, Sagittal T2-weighted spin-echo MR image demonstrates the mass at the posterior epidural space of T9-T11. The mass shows homogeneous high signal intensity. The spinal cord is severely compressed by the mass and shows high signal intensity, suggestive of compressive myelopathy.

$B$, On noncontrast transverse T1-weighted spin-echo MR image, the mass shows heterogeneous isointensity with area of high signal intensity (arrow) at the posterior portion of the mass. The mass extends to the left neural foramen.

C, On sagittal postcontrast T1-weighted image, the mass shows homogeneous strong enhancement. A dural tail sign is also seen (arrows)

$D$, Photomicrograph reveals the formation of large cavernous vascular channels separated by a scant connective stroma. The spaces are lined by a flattened monolayer of endothelial cells $(\mathrm{H} \& \mathrm{E}, \times 100)$.

report on venous hemangiomas. Considering previous reports and our results, we might assume that the cavernous or capillary spinal epidural hemangioma demonstrated a solid hypervascular mass, and the venous or arteriovenous hemangioma could be seen as a cystlike mass. The main purpose of defining the categories was to understand the various MR imaging findings of spinal epidural hemangiomas. Although there were different histopathologic backgrounds that resulted in differ- 

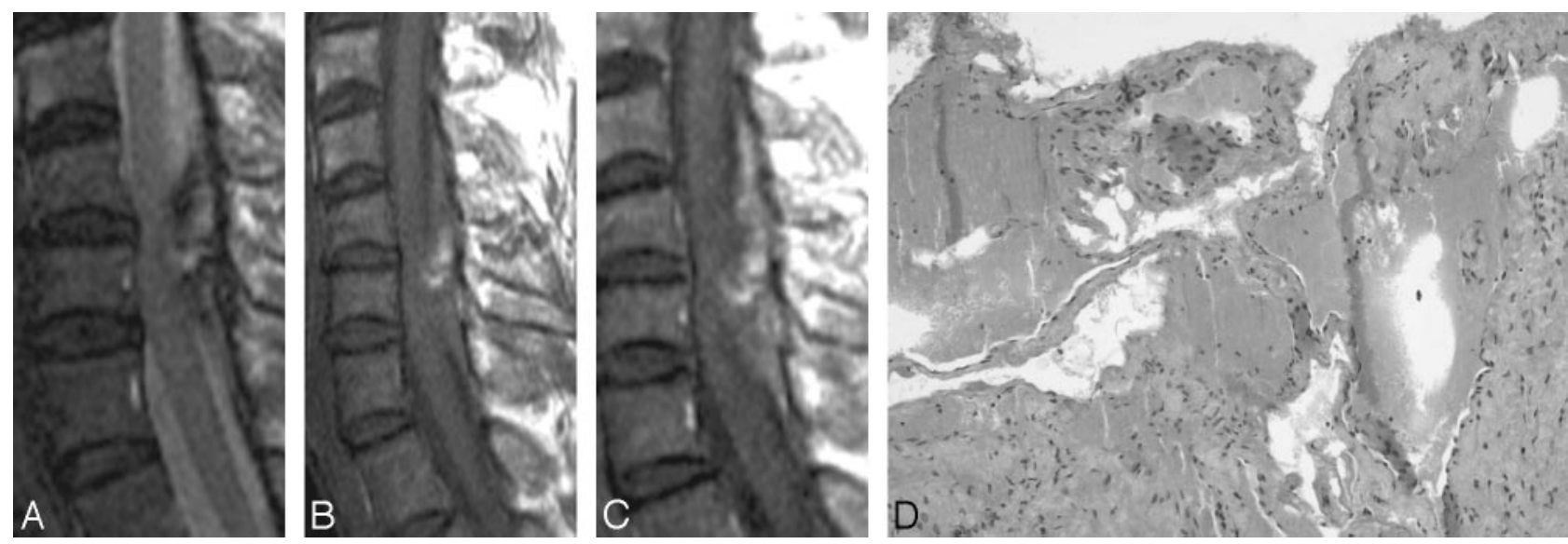

Fig 4. Case 10, an epidural hematoma (type D)

A, Sagittal T2-weighed spin-echo MR image demonstrates an ill-defined lesion with heterogeneous low and high signal intensity at the posterior epidural space of C5-C7.

$B$, On sagittal noncontrast T1-weighed spin-echo MR image, there is high signal intensity in that lesion, suggestive of a hematoma.

$C$, On sagittal postcontrast T1-weighed spin-echo MR image, the lesion shows heterogeneous enhancement.

$D$, On histologic examination, most of the area is an organized thrombus. There are scattered small foci of large, dilated, blood-filled vessels lined by flattened endothelium, which represents a cavernous hemangioma $(H \& E, \times 100)$.

ent MR findings, hemangiomas may have a high risk of intraoperative bleeding, irrespective of the subtype., ${ }^{3,5,9-11}$

In our study, a rim of low $\mathrm{T} 2$ signal intensity was relatively common (57\%, 8/14 cases), as in the previous study. ${ }^{3,8}$ The cause of the low signal intensity rim is uncertain. It may be one of or a combination of 1) the fibrotic capsule itself, 2) the interface between a mass and adjacent dura or posterior longitudinal ligament, 3) a chemical shift artifact created by epidural fat and intratumoral fluid content, and 4) deposition of hemosiderin. ${ }^{3,8}$ In our study, a dural tail sign was not common, but could be seen in 4 (21\%) of 14 cases. It is noteworthy that the arteriovenous or venous hemangiomas were frequently detected as small cystic masses in the anterior lumbar epidural space and induced radiculopathy. In our study, 2 cases demonstrated sudden onset myelopathy caused by an epidural hematoma in the background of a cavernous hemangioma. This finding was similar to that of Carlier et al. ${ }^{22}$

The differential diagnosis of spinal epidural hemangiomas included herniated disks, synovial cysts, granulomatous infections, neurogenic tumors, lymphomas, meningiomas, angiolipoma, pure epidural hematoma, and epidural extramedullary hematopoiesis depending on the MR types, ${ }^{5,8,11,14-19}$ (On-line Table 2). In type A or B, spinal epidural hemangiomas were seen as a cystlike mass with peripheral enhancement. Occasionally, herniated disks, synovial cysts, or granulomatous infections could also be seen as peripheral-enhancing cystlike masses such as type A or B. ${ }^{11,16,17} \mathrm{~T} 1$ hyperintensity may be a clue in the diagnosis of epidural hemangiomas in type A. Unlike the cases of herniated disks or granulomatous infections, which frequently have a thick and irregular rim, our cases showed a smooth and relatively thin rim. The predilection of an anterior epidural space and a person's relatively young age may favor type A or B of a spinal epidural hemangioma to a synovial cyst. The MR features of an epidural solid hypervascular mass such as a neurogenic tumor, lymphoma, meningioma, or angiolipoma may be similar to type C (MR features of a cavernous spinal epidural hemangioma without hematoma). ${ }^{5,8,11,14}$ According to our study, cavernous spinal epidural hemangiomas involved a multisegmental spinal level, and this may provide a clue to an epidural hemangioma. A rim of low signal intensity may also favor an epidural hemangioma to a neurogenic tumor.

Morioka et $\mathrm{al}^{23}$ claimed that the absence of intervertebral neural foraminal widening in epidural cavernous hemangiomas could be a significant clue in the differential diagnosis, with a neurogenic tumor. However, according to a study by Shin et $\mathrm{al}^{8}{ }^{8} 4$ of 5 cases showed neural foraminal widening caused by a cavernous hemangioma. In our study, 6 (66.7\%) cases among 9 with type $\mathrm{C}$ lesions (cavernous hemangioma without hematoma) showed neural foraminal widening. Thus, neural foraminal widening is probably a less helpful feature in the differential diagnosis with neurogenic tumors. Lymphomas or meningiomas frequently show isointense signal intensity on T2-weighted images; thus, this could be a clue in the differential diagnosis because spinal epidural hemangiomas usually reveal high signal intensity on T2-weighed images. ${ }^{5,8}$

Spinal angiolipoma is a rare form of epidural tumor and shows strong enhancement with lobular contours similar to the type C lesions of our study. ${ }^{14}$ According to a study by Provenzale et al, ${ }^{14}$ spinal angiolipoma showed T1 hyperintensity with or without large, hypointense foci on a noncontrast T1-weighted image. According to our study, type $\mathrm{Clesions}$ did not show T1 hyperintensity, unlike angiolipoma. Only ill-defined (type D) or cystlike masses (type A) showed T1 hyperintensity but differed from angiolipoma in their contourlike or cystlike appearance. MR features of pure epidural hematoma or epidural extramedullary hematopoiesis may be similar to type $\mathrm{D} .{ }^{18,19} \mathrm{~A}$ history of a previous traumatic injury, intervention, or coagulopathy may favor a pure epidural hematoma. In rare cases, epidural extramedullary hematopoiesis can manifest as an epidural mass with compression of the spinal cord in patients who are in a chronic anemic state. ${ }^{19}$ In epidural extramedullary hematopoiesis, the signal intensities of the vertebral bone marrow are also changed to diffuse low signal intensities, which provides a clue in the differential diagnosis. In 
summary, a spinal epidural hemangioma could be suspected if there is either a solid hypervascular mass that involves multiple levels of the spine or a cystlike mass with T1 hyperintensity.

Our cases of the type D lesions showed diffuse areas of organized hematoma. However, they also had focal areas of typical cavernous hemangioma. From a histologic standpoint, an organized hematoma is clearly different from a hemangioma. A chronic hematoma is a complex lesion composed of fibrin admixed with degenerated cellular debris in the center with surrounding fibrosis and reactive proliferation of spindle cells. Small capillary proliferation is a minor component of the lesion. In contrast, a cavernous hemangioma consists of collections of large, dilated, blood-filled vessels with a shared wall, which is not found in a hematoma. ${ }^{10}$ For type D, a very low signal intensity on T2-weighted images and a high signal intensity on T1-weighted images strongly suggested a hematoma. However, this was true for only a very brief time. We may have missed other cases with hematomas.

There were several limitations in this study. First, this was a retrospective study. A spinal epidural hemangioma is not a common lesion, so a prospective study might have been impossible. Second, different MR scanners and protocols were used, which was unavoidable because 3 hospitals were referral centers in our country, and patients were referred for an operation after MR imaging at other hospitals. Third, only a small number of cases of the venous and arteriovenous types and none of the capillary type were evaluated in our study because of their rare occurrences. Fourth, 3 radiologists reviewed all the MR images together. It was not possible to evaluate interobserver variability in this study.

In conclusion, spinal epidural hemangiomas can have various MR imaging features according to different histopathologic backgrounds. In addition to common features such as lobular contour, a rim of low T2 signal intensity, and solid hypervascularity, T1 hyperintensity or multisegmental involvement may also be a clue in the differential diagnosis of a spinal epidural hemangioma.

\section{References}

1. Feider HK, Yuille DL. An epidural cavernous hemangioma of the spine. AJNR Am J Neuroradiol 1991;12:243-44
2. Thome C, Zevgaridis D, Matejic D, et al. Juxtaposition of an epidural intraforaminal cavernous hemangioma and an intradural schwannoma. Spine 2004;29:E524-27

3. Badinand B, Morel C, Kopp N, et al. Dumbbell-shaped epidural capillary hemangioma. AJNR Am J Neuroradiol 2003;24:190-92

4. D'Andrea G, Ramundo OE, Trillo G, et al. Dorsal foramenal extraosseous epidural cavernous hemangioma. Neurosurg Rev 2003;26:292-96

5. Minh NH. Cervicothoracic spinal epidural cavernous hemangioma: case report and review of the literature. Surg Neurol 2005;64:83-85; discussion 85.

6. Goyal A, Singh AK, Gupta V, et al. Spinal epidural cavernous haemangioma: a case report and review of literature. Spinal Cord 2002;40:200-02

7. Pastushyn AI, Slin'ko EI, Mirzoyeva GM. Vertebral hemangiomas: diagnosis, management, natural history and clinicopathological correlates in 86 patients. Surg Neurol 1998;50:535-47

8. Shin JH, Lee HK, Rhim SC, et al. Spinal epidural cavernous hemangioma: MR findings. J Comput Assist Tomogr 2001;25:257-61

9. Talacchi A, Spinnato S, Alessandrini F, et al. Radiologic and surgical aspects of pure spinal epidural cavernous angiomas. Report on 5 cases and review of the literature. Surg Neurol 1999;52:198-203

10. Weiss SW, Goldblum JR. Benign tumors and tumor-like lesions of blood vessels. In: Weiss SW, Goldblum JR, eds. Weiss's Soft Tissue Tumors. 4th ed. St Louis: Mosby; 2001:837-90

11. Zevgaridis D, Buttner A, Weis S, et al. Spinal epidural cavernous hemangiomas. Report of three cases and review of the literature. J Neurosurg 1998;88:903-08

12. Saringer W, Nobauer I, Haberler C, et al. Extraforaminal, thoracic, epidural cavernous haemangioma: case report with analysis of magnetic resonance imaging characteristics and review of the literature. Acta Neurochir (Wien) 2001;143:1293-97

13. Rovira A, Rovira A, Capellades J, et al. Lumbar extradural hemangiomas: report of three cases. AJNR Am J Neuroradiol 1999;20:27-31

14. Provenzale JM, McLendon RE. Spinal angiolipomas: MR features. AJNR Am J Neuroradiol 1996;17:713-19

15. Demachi H, Takashima T, Kadoya M, et al. MR imaging of spinal neurinomas with pathological correlation. J Comput Assist Tomogr 1990;14:250-54

16. Apostolaki E, Davies AM, Evans N, et al. MR imaging of lumbar facet joint synovial cysts. Eur Radiol 2000;10:615-23

17. Sandhu FS, Dillon WP. Spinal epidural abscess: evaluation with contrast-enhanced MR imaging. AJNR Am J Neuroradiol 1991;12:1087-93

18. Fukui MB, Swarnkar AS, Williams RL. Acute spontaneous spinal epidural hematomas. AJNR Am J Neuroradiol 1999;20:1365-72

19. Dibbern DA Jr, Loevner LA, Lieberman AP, et al. MR of thoracic cord compression caused by epidural extramedullary hematopoiesis in myelodysplastic syndrome. AJNR Am J Neuroradiol 1997;18:363-66

20. Harrison MJ, Eisenberg MB, Ullman JS, et al. Symptomatic cavernous malformations affecting the spine and spinal cord. Neurosurgery 1995;37:195-204; discussion 204-05

21. Haimes AB, Krol G. Dumbbell-shaped spinal cavernous hemangioma: a case report. AJNR Am J Neuroradiol 1991;12:1021-22

22. Carlier R, Engerand S, Lamer S, et al. Foraminal epidural extra osseous cavernous hemangioma of the cervical spine: a case report. Spine 2000;25:629-31

23. Morioka T, Nakagaki H, Matsushima T, et al. Dumbbell-shaped spinal epidural cavernous angioma. Surg Neurol 1986;25:142-44 